\title{
Design Goals for Playful Technology to Support Physical Activity Among Wheelchair Users
}

\author{
Liam Mason \\ University of Lincoln \\ Lincoln, UK \\ lmason@lincoln.ac.uk \\ Patrick Dickinson \\ University of Lincoln \\ Lincoln, UK \\ pdickinson@lincoln.ac.uk
}

\author{
Kathrin Gerling \\ KU Leuven, e-Media Research Lab \\ Leuven, Belgium \\ kathrin.gerling@kuleuven.be \\ Antonella De Angeli \\ University of Lincoln / University of Trento \\ Lincoln, UK / Trento, Italy \\ adeangeli@lincoln.ac.uk
}

\begin{abstract}
Playful technology has the potential to support physical activity (PA) among wheelchair users, but little is known about design considerations for this audience, who experience significant access barriers. In this paper, we leverage the Integrated Behavioural Model (IBM) to understand wheelchair users' perspectives on PA, technology, and play. First, we present findings from an interview study with eight physically active wheelchair users. Second, we build on the interviews in a survey that received 44 responses from a broader group of wheelchair users. Results show that the anticipation of positive experiences was the strongest predictor of engagement with PA, and that accessibility concerns act as barriers both in terms of PA participation and technology use. We present four design goals - emphasizing enjoyment, involving others, building knowledge and enabling flexibility - to make our findings actionable for researchers and designers wishing to create accessible playful technology to support PA.
\end{abstract}

\section{CCS CONCEPTS}

\section{- Applied computing $\rightarrow$ Computer games;}

\section{KEYWORDS}

Accessibility, Games, Integrated Behavioral Model

\footnotetext{
Permission to make digital or hard copies of all or part of this work for personal or classroom use is granted without fee provided that copies are not made or distributed for profit or commercial advantage and that copies bear this notice and the full citation on the first page. Copyrights for components of this work owned by others than ACM must be honored. Abstracting with credit is permitted. To copy otherwise, or republish, to post on servers or to redistribute to lists, requires prior specific permission and/or a fee. Request permissions from permissions@acm.org.

CHI 2019, May 4-9, 2019, Glasgow, Scotland Uk

(C) 2019 Association for Computing Machinery.

ACM ISBN 978-1-4503-5970-2/19/05 ..\$15.00

https://doi.org/10.1145/3290605.3300262
}

ACM Reference Format:

Liam Mason, Kathrin Gerling, Patrick Dickinson, and Antonella De Angeli. 2019. Design Goals for Playful Technology to Support Physical Activity Among Wheelchair Users. In CHI Conference on Human Factors in Computing Systems Proceedings (CHI 2019), May 4-9, 2019, Glasgow, Scotland Uk. ACM, New York, NY, USA, 12 pages. https://doi.org/10.1145/3290605.3300262

\section{INTRODUCTION}

Regular physical activity (PA) is known to be important for general health and wellbeing [1]. However, it has been shown that many of the estimated 65 million manual wheelchair users in the world do not achieve recommended levels of activity [15], and miss out on the associated health benefits, such as reduced physical pain and a lower risk of depression [40]. The potential of playful technology to motivate and support PA among non-disabled people has been addressed by the research community: for example, systems such as the Nike Fuelband [3], or video games like Dance Dance Revolution [26]) encourage significant PA. However, little work has considered the design of technology to support PA among individuals with mobility impairments, and manual wheelchair users in particular. Existing work in this space either focuses on expert users (e.g. activity monitors for wheelchair athletes [10]), movement-based games with an emphasis on player experience (such wheelchair-controlled video games for older adults [20] or young people with mobility impairments [45]). This existing work does not directly address the needs of the wider manual wheelchair user, from the perspective of supporting PA levels within current health guidelines.

Our work addresses this gap through an exploratory enquiry into the role of playful technology to support PA among manual wheelchair users, and identifies and addresses facilitators and barriers within the context of mobility impairment. We follow a mixed-methods approach that combines interviews with a survey study, and we build on the Integrated 
Behavior Model (IBM), a general theory that posits that intentions are the strongest predictor of human behaviour. We use this framework to guide an exploration of wheelchair users' perspectives on PA, technology, and play, and present initial findings from in-depth interviews with eight manual wheelchair users which show comprehensive engagement with PA, but also reveal a number of challenges, e.g., stigma, environmental barriers and knowledge. We then draw from these results to develop a questionnaire, which was widely deployed online, and received 44 responses; analysis revealed that wheelchair users' PA behavior is positively influenced by the anticipated experience, but subject to various environmental barriers. Furthermore, survey results support interview findings which demonstrate an interest in technology and engagement with games. Drawing on both sets of results, we propose design goals to be considered in the development of technology to support PA among wheelchair users, and reflect on the role of games and play in this setting.

Our paper makes the following contributions: (1) We provide the first in-depth exploration of the role of technology in the context of PA among wheelchair users, and (2) we provide an example of how the IBM can be leveraged in exploratory HCI research. (3) We provide design goals to inform the creation of playful technology to support PA among wheelchair users, drawing attention to factors relating positive perspectives on PA, play, and accessibility.

\section{BACKGROUND}

In this section we summarise existing research on PA among wheelchair users, predominantly from the perspective of sport science and psychology; we also examine existing technology which has been designed to support PA for wheelchair users. We furthermore provide an overview of the Integrated Behavior Model (IBM) as theoretical foundation for our work.

\section{Physical Activity and Wheelchair Use}

Given the importance of PA, and often low levels of engagement by wheelchair users, some existing work has sought to identify barriers to participation (e.g. [28]). Results show that some issues generalize to all audiences, e.g., limited time, or financial cost; however, others are more specific to wheelchair users, such as the fear of stereotyping [44]. Focusing on young users, Buffart et al. [8] revealed barriers which included attitude, motivation, existing injury or fear of developing injuries, limited facilities, and a lack of information or knowledge. Facilitators included associated fun, opportunity for socializing, and improved health and fitness. Shields [37] broadly supports these outcomes, but also highlights the importance of logistical and psychological barriers. Wadey and Day [42] considered PA among adult users who had lost a limb, and identified a number of themes, including wellbeing (personal, social, and physical), inspiration, self-presentation, experience of PA, knowledge, environment, and organisational functioning. Williams et al. (2014) identified eight interrelated concepts as barriers, benefits and/or facilitators of PA: wellbeing, environment, physical body, body-self relationship, physically active identity, knowledge, restitution narrative and perceived absences. Generally, previous work points at complex interaction between individual, social and environmental factors that can either support or hinder participation [28]. These works all highlight a need for a better understanding of the design space to facilitate the development of technology to support PA among wheelchair users.

\section{Technology to Support PA Among Wheelchair Users}

Previous work has explored different technologies to facilitate and support wheelchair users' engagement with PA, with one stream of work focusing on activity tracking, and the other exploring the value of movement-based play.

Tracking Technology. Carrington et al. [10] explore the use of Fitbit ${ }^{T M}$ with wheelchair athletes, and discuss low adoption rates due to accessibility challenges. In another study using bespoke devices, the authors demonstrate that participants were excited, and perceived the tracking data as useful for improving their own skills [9]. Following a similar line of work, Malu and Findlater [27] highlight widespread accessibility challenges with existing technologies, and demonstrate the desire of participants to engage with activity tracking, underlining the potential utility of such solutions to users.

Playful Technology. While commercially available exergames accessible for this audience are scarce [29], a number of researchers have explored movement-based play for wheelchair users. For example, toolkits such a KinectWheels [19] and Mokey [14] facilitate the integration of wheelchair-based game input. Focusing on the potential of movement-based play to encourage PA, Hicks et al. [23] created games for young wheelchair users, concluding that participants were very enthusiastic about the concept. Astrowheelie [12] and GameWheels [35] are further examples of gaming systems specifically designed for wheelchair users; in both cases, the authors concluded that participants had fun while exercising. However, these games were designed to encourage movement rather than more intense PA, and it remains unclear how they align with the general values and needs of wheelchair users.

\section{Integrated behavioral Model (IBM)}

The IBM combines the theory of reasoned action (TRA), and the theory of planned behavior (TPB) into a single model [30]. TRA asserts that the most important determinant of a 
Design Goals for Playful Technology to Support Physical Activity Among WGiekl2här, Nkeys4-9, 2019, Glasgow, Scotland Uk

person's behavior is behavioral intention (comprising attitude and subjective norms). Later, in an effort to account for factors outside of the individual's control, Ajzen proposed TPB as an extension which added an additional construct; perceived behavioral control [2]. The IBM is a general theory of behavioral prediction that is applicable to the understanding of any given behavior. Similar to TRA and TPB, the IBM proposes that intentions (attitudes, perceived norms, and self-efficacy) are the primary determinant of behavior. However, it also integrates four additional components that directly affect behavior: knowledge, salience, environmental constraints, and habit [30], giving the best coverage of factors relevant to our research setting. Attitudes are split into experiential (feelings) and instrumental (beliefs) which refers to an individual's overall perception of a particular behavior. For example, if a wheelchair user believed that doing regular exercise would result in improved physical health, and that health is a good thing, then they would have a positive instrumental attitude toward PA. Perceived norm reflects beliefs regarding other people's attitudes (injunctive norms) and behavior (descriptive norms) in relation to a behavior. Personal agency refers to an individual's perceptions of their ability to engage in a behavior, split into self-efficacy (an individual's belief in their effectiveness in performing a task as well as by their actual skill) and perceived control (an individual's perceived amount of control over behavioral performance).

Together, these factors influence the intention to engage, which itself influences actual behavior. The goal for researchers is to understand which of the behavioral determinants are most strongly related to intention, and to use that knowledge to develop interventions which influence health behaviors. De Angeli et al. [13] demonstrated how the IBM can be used as a method of eliciting user requirements, stating that it provided rich information on motivations and barriers to activities for older people, and concluding that the IBM was efficient, reliable and easy to link to design thinking . Our work also leverages the IBM as a theoretical framework for further exploration of perspectives of wheelchair users on $\mathrm{PA}$, and the potential for the use of technology in this setting.

The IBM has been extensively applied in empirical research; for example, it has been used in health care to promote HIV prevention [24], understand binge drinking [6] and, (most relevant to this study) to facilitate PA among children [5]. Interestingly, even though the IBM has previously been applied to PA and tested among several demographics, it has not yet been used with wheelchair users. Thus, our study contributes to the growing literature of theory-based determinants of PA among wheelchair users while paying special attention to the role of technology and play in this setting.

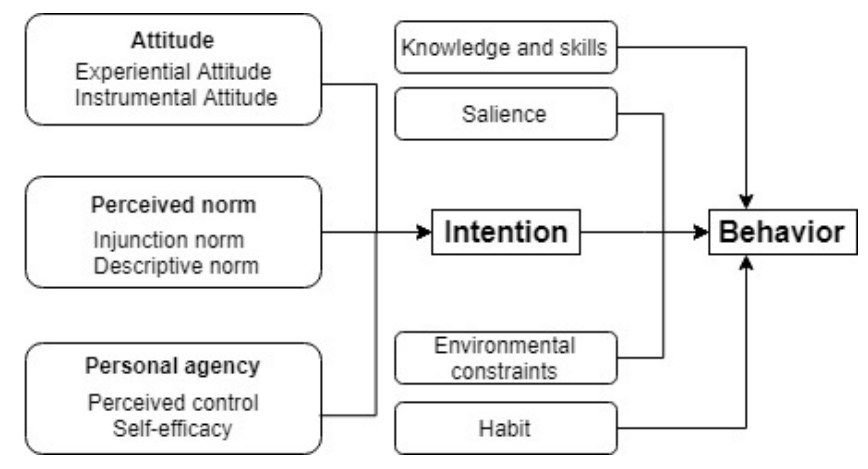

Figure 1: Integrated behavioral Model (IBM)

\section{STUDY: HOW DO WHEELCHAIR USERS ENGAGE WITH PA AND TECHNOLOGY?}

Following the protocols of the IBM, we carried out an initial interview study which we then used to inform a more extensive online survey. Together, these explore the potential of playful technology to support PA among wheelchair users. To this end, we aim to answer two research questions (RQs):

RQ1: What perspectives (e.g. attitudes) of wheelchair users could be used to design of technology which supports PA?

RQ2: What is the perceived potential of playful technology to support PA among wheelchair users, and what are the associated opportunities and challenges?

This study used mixed methods for gathering data, and was conducted in two phases. Qualitative open-ended elicitation interviews were the foundation of the first phase of the research: These were conducted to empirically confirm the IBM's constructs, and to design a quantitative online survey. Using interviews to elicit information from wheelchair users allowed us to develop appropriate items for each of the model constructs. Montano describes that the qualitative interview stage is considered essential to the development of a survey that would be culturally appropriate, and relevant to the populations being sampled [30].

\section{Part 1: Interview Study}

In this section we present results from our initial interviews with manual wheelchair users, which address both of our research questions concerning PA and the use of technology.

Participants and Procedure: Eight participants (2 female, 6 male; age ranging from 20 to 61 years old, mean 33.8) were recruited. We used local advertising, and a number of social media channels. All participants were manual wheelchair users (two to 35 years of use, mean 11); four lived in rural 
Table 1: Frequency and valence of IBM dimensions

\begin{tabular}{lll} 
& Positive & Negative \\
\hline Attitude & 65 & 10 \\
\hline Perceived Norm & 95 & 113 \\
\hline Personal Agency & 75 & 128 \\
\hline
\end{tabular}

areas, three were from urban areas, and one participant reported moving between rural and urban life. Six participants were based in the UK, one in Latvia, and one in Norway. All participants lived with family, partners or flatmates, and had a broad range of experience with technology and games.

Semi-structured interviews were conducted in person, at a suitable location chosen by the participant (3), or via means of remote communication (5). At the beginning of each interview, participants were provided with information about the study, and signed consent forms. Participants were asked to reflect on their experiences with $\mathrm{PA}$, through interview questions based on the IBM constructs and playful technology use. This included questions such as: "how do you feel about the idea of being physically active?"; "How much control do you have over physical activity?"; and "Can you think of any experiences you've had with physical activity and technology?". The research protocol was approved by the ethics committee of University of Lincoln.

Data Analysis: Data were analyzed using Thematic Analysis following both a deductive and inductive approach, as laid out by Braun and Clarke [7]. All the statements relevant to constructs of the IBM that describe behavioral intention were extrapolated in a deductive manner. Codes were constructed according to the IBM, and gathered into five families labelled using the original categories of the model (attitude, perceived norm, and personal agency). Each code family was further enriched with specific codes (e.g., "Defying stereotypes") and the emotional valence of each statement was assessed +/. An inductive approach was used to craft themes related to the role of playful technology, in the context of PA and wheelchair use. In order to ensure validity, $40 \%$ of the data were double coded.

Results: We first report results of our deductive analysis, which is based on factors of the IBM (see Figure 1), and then provide an overview of the inductive analysis which focuses on technology sub-themes including preferences for game themes and technologies, subversive player behaviour, accessibility, and negative attitudes toward games.

Attitudes. Generally, participants showed positive feelings towards experiential and instrumental attitudes in relation to PA, elaborating on physical and mental wellbeing, fun, and enjoyment. For example, one participant commented that he was "Feeling like I'm actually able to do something again" [Referring to wheelchair basketball] (P4), "I feel happier" (P7) and "I feel good" (P1). Participants also pointed out several benefits of performing PA, e.g., "I like the idea that I am not putting on weight", "Keeping joints moving [helps] back pain as well" (P2). One participant hoped that PA would help "gaining physical strength maybe and catching up to normal people in terms of going out and do stuff" (P4). Participants also mentioned the possibility of meeting new people and making friends while performing PA. However, negative attitudes were also revealed, e.g., experiencing social anxiety and regret for not participating, or believing that PA might result in injury, ranging from "Get blisters" (P4) to more serious concerns such as worsening their disability.

Perceived Norms. Social pressures to perform or not perform a particular behavior were highly relevant to participants' perspectives on PA. Expectations of other stakeholders were a sub-theme with both negative and positive implications: participants mentioned how other people motivated them to participate in PA, with family being the biggest influence. However, family involvement could also be demotivating; for example, others being overly worried, or showing a lack of understanding that led to intrusion (such as having unrealistic expectations about weight loss). Apart from the relevance of family, one participant described how they built a connection with their physiotherapist that contributed to participation in PA. In contrast, participants did not show interest in the PA routines of others, such as friends or family (descriptive norms).

Our data suggest that role models have a strong influence on participants becoming physically active; they reported numerous instances where the observation of disabled athletes inspired further engagement (for example, taking up wheelchair basketball after watching it in the Olympics). A recurring theme relevant to norms was the desire to defy stereotypes, and, by extension, disability. Participants explained that they were motivated to carry out PA to prove their abilities to non-disabled people, e.g., "There's a few people that see me and think because I'm disabled I get tired [...] I get out and go." (P3) and "I do like to piss people off, surprise them, oh you play wheelchair basketball, ye now fuck off!" (P6). However, interview results also revealed that participants were aware of performance differences. For example, one participant would not want to share activity data because they expected non-disabled users to achieve better results.

Personal Agency. Most (7) participants reported PA routines that suggested high perceived self-efficacy; for example, one participant showed strong awareness of their own 
skills to the point where they could coach other wheelchair users confidently. However, some participants felt that their disability limited access to certain elements of PA, such as participation in competitive sports. Our data also shows that perceived agency is strongly influenced by access to specialized equipment. Some participants reported keeping a spare wheelchair to maintain mobility at all times; in contrast, other participants were concerned about affordability and storage space. Likewise, a recurring barrier was a lack of spare time (or lack of control thereof): participants reported that other aspects of their lives limited opportunity to engage in PA (work or family life). In the context of agency and selfefficacy, participants did express an interest in technology that supported this aspect.

Salience. All participants were aware of the importance of PA, and regularly engage in it, though there were a variety of different responses given as to why it was important to do so. Participants often referred to health benefits, but they also mentioned psychological factors, such as being social, and avoiding depression (instrumental attitude). As previously mentioned, one participant engaged in PA to set a good example for his children.

Knowledge. Participants were knowledgeable in terms of activities they already engaged in. For example, one participant did not only play wheelchair basketball, but was also involved in coaching wheelchair athletes and non-disabled persons. However, when it came to new activities, participants often struggled to determine whether it would be possible to gain access; "I have to inquire about any activities I want to do [...] which might be discouraging to even try" (perceived control).

Habit. Routine was a strong theme that was apparent in data. Group activities such as wheelchair basketball took place on set days, participants reported going to the gym on set days, and one participant mentioned regularly pushing along the beach after dropping off their son. There was no evidence of spontaneous PA, which requires no thought or planning. The only related activity with no set occurrences was movement-based video game play, mentioned by three participants (involving Nintendo and Sony console systems).

Environment. A number of environmental barriers need to be considered, spanning the natural, built (including infrastructure), and social environments of participants. In terms of the natural environment, weather was a significant constraint. Rain and snow were causes of concern; however, one participant commented that hot summer months were draining. Two participants lived in countries with much colder climates (Norway and Latvia). One of the participants used this to their advantage, taking part in winter sports, while it caused problems for the other: "Winters and cold weather definitely restrict me to be more active and outgoing, as it's hard to move around heavy snow or very low degrees, when it's extremely cold" (P7).

Infrastructure and the environment were mentioned by participants, half (4) of whom had cars. This allowed them to not only access the locations where they perform PA, such as the gym or clubs, but to also facilitate independent living in general. One participant said that they can go anywhere "as long as I can get to my car" (P8), while another stated that a nearby city was much more accessible for a wheelchair user because "around here you don't even get bloody footpaths really." (P6). The participants who did not drive mentioned more barriers to transportation, such as expense, inconvenience of waiting times, getting home in the dark, and non-accessible areas such as stairs or roadworks. All participants who were part of a club reported that they liked playing in a social environment. Others described how physiotherapists, coaches or groups aid in PA. Two participants did not engage in social sports, and exercised on their own. These participants appeared to be the least active, suggesting that they lack knowledge to perform exercises that would benefit them.

Perspectives on Technology and Play In this section we report findings from our inductive analysis, which reveals perspectives on technology, games and play. All but one participant reported playing games, although time spent playing games varied. Four participants mentioned using technology to facilitate PA. Two participants described using the Nintendo Wii (and the game Nintendo Wii Fit in particular) to engage in PA, but also mentioned that use had declined over time. Through analysis, we crafted four sub-themes from the data: Preferences for game themes, genres and technologies, subversive user behavior, accessibility barriers, and negative attitudes towards games.

Preferences for game themes, genres and technologies. Participants described specific game themes, genres and technologies they disliked, with little overlap between each other. For example, participants mentioned genres they were not interested in, "a warrior-based fighting game that doesn't really interest me" (P4), "I like playing simulation games most.", and commented on new technologies, "Augmented reality doesn't interest me at all" (P1) along with features "Newest multiplayer PC games" (P5). Also relating to genres, participants mentioned the appeal of certain types of games as a means of escaping from the reality of their limited mobility, e.g., "I am not able to play tennis, but I can play a Wii Game Console tennis." (P7). The sub-theme of brand loyalty was strong, with almost all participants mentioning some form 
of preference such as not using the Kinect because it is associated with the Xbox, or only using Apple devices.

Subversive user behavior. There were several instances which suggest that users may intentionally undermine game mechanics related to PA: some participants described how they cheated while playing games to avoid activity. For example, "[You only make an effort] until you actually realise you can sit on a sofa and just wiggle the control." (P5), "but I cheat using two things [stylus pens for input]" (P4). Likewise, one participant, when describing their weight watchers system, mentioned that they do not consistently track their progress, ultimately making the system redundant.

Accessibility barriers. Several participants mentioned accessibility issues, spanning both practical barriers and wider perspectives on inclusion. In terms of practical barriers, participants described difficulties with technology, such as malfunctions: "it would phone the police when you're in a wheelchair pushing it [using a smart watch]" (P6), or inaccessible user interfaces "so I don't have a spare hand available to do it, and that is just asking me to drop my phone" (P4). Participants were often advocates for wider inclusion. One participant stated that they would not play a game that did not try to include accessibility features, stating that "when I hear someone else who can't try it out and the companies don't do anything I am like no, I don't want to do it either" (P1).

Negative attitudes towards games. While (5) participants reported playing games themselves, three of them saw games in a negative light, commenting that it was just "pushing buttons" (P6), with some suggesting that games were not comparable to sports. One participant criticized others for playing Pokemon Go too much, with another not playing games because he did not want to influence his son. At the same time, our data showed that other mobile games (e.g., played on an iPad) were perceived in a more positive light.

\section{Part 2: Online Survey}

Survey Description and Procedure. Based on interview outcomes and the IBM, a questionnaire with a total of 62 questions was developed, split into three main sections: (1) a section that explores demographics (age, wheelchair use, engagement in PA, and technology use). Sample questions include "How long have you been a wheelchair user?" and "I would consider myself very physically active". (2) measurement of the theoretical model constructs of the IBM using 5-point Likert scales (attitudes (8), perceived norm (11), personal agency (17)), with items such as "I enjoy PA", "I am motivated to engage in PA by defying stereotypes", and "I have a high amount of control in being physically active". (3) a final part that explored perspectives on technology in general. and games in particular. through a number of closed and open-ended questions. Sample questions include "I like to play video games", "I think digital technology could facilitate PA for me", and "Are there any specific barriers you encounter when using technology with PA ?".

IBM items were presented in random order. Participants were shown different questions based on their answers in the final section; e.g., if a participant was strongly opposed to the idea of leveraging games to engage in PA, they would be shown questions further exploring this perspective. Pilot tests of the survey were conducted with eight non-wheelchair users (due to population size), leading to some questions being re-worded or removed in order to ensure that language used in the survey was understandable, and survey length was appropriate (between 10 and 15 minutes). The final survey was made available online using Qualtrics, access information was distributed through local wheelchair organizations and social media (Facebook, Twitter, Reddit). At the start of the survey, participants were presented with a consent form, and indicated their agreement to complete the survey. They were then directed to the survey questions, which they were able to complete at a time and location of their choice.

Data Analysis. The survey received a total of 75 responses, of which 44 were deemed complete and included in our analysis. The data were analyzed using SPSS Version 16 statistical software. Open-ended questions were analyzed using inductive Thematic Analysis, using NVivo 12.1.

Results. In this section, we give an overview of the survey results. First, we provide in-depth descriptions of the respondent sample to put following quantitative and qualitative data in perspective. We then report the results of the IBM along with outcomes of qualitative analysis of open-ended parts of the survey.

Characteristics of Respondents. Gender was reported as male (23), female (18), other (1) and prefer not to say (2). Participant age ranged from 18 to 64 (18-24 (14), 25-34 (14), 35-44 (8), 45-54 (2) and 55-64 (6)). All responses were provided by participants living in western societies including Europe, the USA, and Australia. A total of 41 respondents were current wheelchair users. Wheelchair use ranged from less than six months to more than ten years (less than 6 months (2), 6 months to a year (4), one to two years (11), two to five years (10), five to ten years (5) and 10+ years (9)), with amount of use being varied (all the time (26), most of the time (11), moderately (2) and very little (2)).

On a 5-point Likert scale participants reported their perceived level of PA as $(M=3.41, \mathrm{SD}=1.32)$, with the sample as a whole reporting a high amount of enjoyment from PA 
(M 4.14, SD .88). Interestingly, spontaneity $(\mathrm{M}=3.66 \mathrm{SD}=$ $1.18)$ and routine $(\mathrm{M}=3.67 \mathrm{SD}=1.18)$ shared very similar results. Participants mentioned a wide variety of activities that they participated in, and which they considered to be PA. These were split into three categories: Everyday activities, e.g., showering, shopping and commuting (17); recreational, such as basketball, squash and archery (20); and health, such as gym, yoga and physiotherapy (24). Some activities were placed in multiple categories (e.g.going to the gym for fun).

Participants' engagement with games was relatively high $(\mathrm{M}=3.67, \mathrm{SD}=1.44)$, mentioning a wide range of game genres with the most common being role playing, action and puzzle. Delving deeper into the use of video games and technology, participants reported a preference for playing video games over other activities $(\mathrm{M}=2.97, \mathrm{SD}=1.40)$, cheating in video games $(\mathrm{M}=2.93, \mathrm{SD}=1.38)$ and accessibility issues $(\mathrm{M}=3.03 \mathrm{SD}=1.46)$, interestingly all had a high standard deviation suggesting varied views among participants. Loyalty to platforms $(M=3.46, S D 1.37)$ and importance of game theme $(M=3.84, S D=1.19)$ reported on a whole a higher response. Participants reported a positive outlook on the potential of using technology to support $\mathrm{PA}(\mathrm{M}=3.71, \mathrm{SD}=$ 1.06). However they also reported a low use of technology in PA $(M=2.91, S D=1.16)$. Participants showed a high level of interest in the potential of engaging in PA with video games (3-point scale Yes (12), maybe (12), no (3)) respondents also reported that they had a preference for games that included social interaction $(\mathrm{M}=4.20, \mathrm{SD}=.73)$.

It was not only important to understand whether participants would engage with technology in PA, but to also to determine what sort of engagements would be appealing. Participants reported using a wide range of technology: Smart phone (38), personal computer (33), tablet (25), Fitbit/fitness trackers (15), Nintendo Wii (11), Nintendo Switch (8), Xbox Kinect (8), VR systems (5) and PlayStation Move (3). Some also mentioned a few devices that were not listed such as Nintendo DS, Gameboy and Sony PSP. Participants also reported a sufficient amount of space available to engage in $\mathrm{PA}$ at home (no room (2), can engage in basic wheelchair movement (20), extensive movement possible (7)).

\section{Analysis of IBM constructs}

An overall index was computed for each dimension of the IBM by averaging scores to related items (Table 2). Linear regression was used to estimate the relationship between the IBM indexes and the perceived level of PA. Due to the limited size of the sample, a two-step procedure was applied. In the first step, three separate regression analyses were performed one for each construct. Responses to the question "I would consider myself physically active" were entered as the dependent variable because it allowed us to estimate the relationship between the constructs of the IBM (predictor variables) and the self-reported level of PA, exploring which IBM constructs are most relevant to wheelchair users engaging in PA [5]. For each item, a preliminary inspection was performed to ensure no violation of the assumptions of normality, linearity and multicollinearity. Experiential attitudes, descriptive norms, and perceived control were determined to be the most significant contributing factors for each construct. Therefore, in the second step they were entered as the three predictors of the analysis. It resulted in a statistically significant equation $(\mathrm{F}(3,36)=11.1, \mathrm{p}<.001)$, $\mathrm{R}^{2}=.50$, standard error .95 . Experiential attitude made the strongest positive contribution with a standardized coefficient beta score of .55 , followed by descriptive norm (.23), and perceived control (.09).

Table 2: Mean scores and Standard Deviations for IBM constructs rated on 5-point Likert scales (5=highest).

\begin{tabular}{|l|l|l|}
\hline Predictor variables & M & SD \\
\hline Experiential Attitude & 3.84 & .92 \\
\hline Instrumental Attitude & 3.87 & .44 \\
\hline Descriptive Norm & 3.92 & .90 \\
\hline Prescriptive Norm & 3.51 & 1.17 \\
\hline Self-efficacy & 3.34 & .59 \\
\hline Percieved Control & 2.65 & .63 \\
\hline
\end{tabular}

\section{Qualitative survey findings}

Here, we give an overview of prominent themes discovered through qualitative analysis of participants' open-ended feedback, focusing on elements of technology use and perspectives on games, in order of importance.

Accessibility: A total of 27 responses relating to the theme of accessibility were reported. Firstly, participants specifically mentioned that their disability affects their use of technology and games, with three participants specifically describing the issues they had previously experienced with the popular location-based game Pokemon Go. Location tracking was also mentioned with one participant expressing that they would like a system that allowed for accessible routes. One participant even used a combination of Strava and Garmin (location based fitness tracking) because a Fitbit was not suitable for them. Controllers were also mentioned with one participant stating that "Motion controllers are too demanding" and another wanting "More adaptive controllers". The accessibility of portable devices was also mentioned "hard to use whilst moving [smartphone]", one participant suggested that it "has to be easily carry-able", with another preferring portable devices because consoles take too long to set up. 
Other accessibility issues such as concentration were mentioned, along with motor issues impacting use of controllers.

Knowledge: Participants also mentioned that they lacked knowledge about how to exercise; "I want to know how to exercise on a wheelchair. I will be member of a gym soon, but I don't know what and how to exercise". Interestingly, one participant compares and learns techniques from other wheelchair users in their town. There were also cases of participants not knowing how to exercise with technology; "technology is the way forward, but difficult to understand for me".

Identity: Similar to the interviews, stigma was a prevalent theme with many negative comments collected. The indicated disapproval of video games and/or technology was attributed to being "too old", "old fashioned" or "a girl". There were also examples of participants perceiving games as a waste of time, or a distraction, and something that they don't want to "play with children". Regardless there were examples of participants finding games fun with one example of a participant stating "it makes me work harder to better myself".

Interest: A lack of interest was demonstrated by a few participants; one noted that they used to track their fitness on a smart phone, but quickly lost interest. One participant stated they "would rather go outside", and replied "better things to $d o$ " in response to why they don't like video games. However a few participants expressed an interest in trying.

\section{Summary of Key Findings}

RQ1: Attitudes and PA participation. The results show that experiential attitude (feelings) was the highest reported construct of the IBM that contributes to PA engagement across interviews and surveys, suggesting that wheelchair users engage in PA because it is enjoyable. Descriptive norms (social identity) was the second highest construct. However, results from interviews and surveys offered different perspectives, with interview responses showing no interest in what other activities others were doing. Finally, results show that perceived control was the least contributing factor, suggesting that control beliefs do not highly influence PA engagement. Qualitative results support elements of the IBM. Most prominently, the theme of knowledge directly links to the corresponding IBM construct; results show that participants struggled to determine if they could partake in new activities (interviews), or how to be physically active at all (survey). Additionally, both data sets showed desire for learning from others; however, many survey respondents were limited to online communities. Finally, the concept of routine was strongly evident across all participants, with PA participation often taking place at fixed times and locations.
RQ2: Technology use and games. Results show that participants use technology, but experience access barriers particularly in the context of leveraging technology to support PA. Considering the use of games, responses suggest high levels of familiarity with games, along with regular participation in play, but also revealed stigma that some individuals associate with playing games. There was wide variety in gaming preferences (e.g., genres) and platforms (e.g., mobile, consoles, or PC), suggesting a need for tailored solutions.

\section{DESIGN GOALS}

We summarize our findings into four main design goals to be addressed in the design of (playful) technology to support PA among wheelchair users. Each design goal is accompanied by examples of commercially available technology and/or games that illustrate how these goals could be realized positively (or avoided in the case of anti-patterns [4]).

Design Goal 1: Emphasize Enjoyment. Our results show that the anticipation of positive experiences is a strong predictor of wheelchair users' engagement in PA (Relevant IBM Construct: Attitude - Experiental Attitude). To reflect this perspective, technology to support PA should first and foremost focus on the facilitation of enjoyable experiences, rather than potential health benefits (instrumental attitude), or aspects external to the activity. Medicalizing PA, and prioritizing health benefits of exercise over enjoyment is a common pitfall when designing for audiences with special needs [36] illustrate this challenge when addressing older adults). Playful technology in particular aligns with this goal, but could potentially be undermined by creation of primarily 'serious' or 'persuasive' games.

Reflection on games that integrate PA while prioritizing enjoyment. Research by Mueller et al. (e.g., [[32], [43]) comprehensively illustrates how playful technology and games can be leveraged to increase the enjoyment of PA, strongly emphasizing the experience of the user. Guidelines that stem from this line of work (e.g., [31]) provide valuable insights that can help create enjoyment-centric rather than disability-focused systems. Likewise, there are many examples of commercially available games following this paradigm, e.g., the VR music game BeatSaber [17] which offers engaging game mechanics, and provides PA as a beneficial byproduct.

Design Goal 2: Involving Others: Our results suggest that involving other parties (e.g. friends, or non-disabled individuals) appeals to wheelchair users, but also comes with challenges that require careful consideration (Relevant IBM Construct: Perceived Norm - Descriptive Norm). For example, simply including multiplayer game modes does not 
necessarily facilitate the involvement of other players adequately. Designers should consider that some players may feel uncomfortable sharing their performance with others (especially in direct comparison with non-disabled players), whereas other players may be excited about activities that give them an opportunity to showcase accomplishments and learn from others. This is in line with previous work exploring competition between wheelchair users with peers and non-disabled people [21], demonstrating that competition and comparison can, depending on implementation, either expose vulnerability or contribute to empowerment. Thus, features involving multiple players could be made optional.

Reflection on playful technology implementing social features in innovative ways. Games have a long history of social features, ranging from direct involvement of multiple persons in gameplay to elements such as shared scoreboards. Examples of design strategies interesting in this setting include games such as New Super Mario Bros. [34] (co-located multiplayer mode with asynchronous player roles), to applications that support engagement in PA over a distance [32]. Additionally, it might be valuable to explore gamified fitness applications such as Fitocracy [16] that enable users to find small online fitness groups, which is also valuable for playful technology with small target audiences. From a technical perspective, our findings further support the implementation of crossplatform solutions that are common in game development, thereby enabling players to use different technical platforms.

Design Goal 3: Build Knowledge: Playful technology to support PA should enable wheelchair users to build and extend their knowledge of suitable PA routines, addressing the self-reported gap identified in our survey (Relevant IBM Construct: Knowledge). Previous research has demonstrated effectiveness in implicitly teaching new skills (e.g. scaffolding in puzzle games [25]). When developing playful technology to support PA among wheelchair users, gradually extending the mechanics of the game could support players wishing to expand their knowledge of exercise. Likewise, careful scaffolding could counteract fears of injury that were reported by some study participants. In this context, designers and developers could also reflect on how playful technology facilitates the transfer of skills and knowledge into daily life, supporting players wishing to explore other activities.

Reflection on games that facilitate scaffolding and help players build knowledge. Game design has a long history of supporting scaffolding, e.g., through tutorials and gradual introduction of game elements that can be observed in many commercially available games [41]. For example, the first-person shooter Portal [11] is a prominent example that applies stepby-step introduction of new functionality to facilitate learning. This approach could directly be applied to knowledge about PA when integrated as main mechanic that gradually increases in complexity. However, designers need to be aware that successful PA participation is not just a matter of knowledge of how to exercise, but also gradual skill building that is particularly relevant in the context of players who recently transitioned to wheelchair use.

Design Goal 4: Enable Flexibility: There are a number of ways in which flexibility is important when creating technology to support PA. Firstly, flexibility is pertinent to interface design; i.e., hands-free operation may be important to some users, so as not to impair operation of their wheelchair. Secondly, users require control over the time and place at which they engage with PA, e.g., to negotiate environmental barriers (Relevant IBM Construct: Personal Agency - Perceived Control). Users also require flexibility in how they blend PA with existing routines (Relevant IBM Construct: Personal Agency - Habit). We note that many participants reported habitualized PA routines that leave little room for spontaneity; for example, attending the gym for a number of hours, but not engaging in other activities during the week (due to access barriers). Game-based PA interventions could thus potentially support flexibility by encouraging PA participation at other times and locations; for example, suggesting shorter, frequent, and more accessible exercises to counteract sedentary lifestyles [22].

Reflection on flexible playful technology in the context of PA. The concept of casual exergames [23] [18] - games that require PA and can be played in short chunks throughout the day - demonstrates that games can be leveraged to facilitate continuous participation in PA. However, developers need to be mindful that core mechanics also need to show flexibility to avoid access barriers. For example, games such as Pokemon Go [33] require players to access certain areas, which is problematic as the game does not account for environmental accessibility barriers such as stairs. This could be addressed through concepts such as accumulated-context exergames, where player activity is tracked throughout the day and asynchronously mapped onto the game world, offering choice in terms of time and location of PA [39].

\section{DISCUSSION}

This paper explores wheelchair users' perspectives toward physical activity, technology, and games, leveraging the IBM as a theoretical model. Here, we discuss more nuanced findings with focus on wheelchair use and PA, pitfalls in the context of playful technology to support PA, and we reflect on the application of the IBM as HCI research tool. 


\section{Playful Technology, PA, and Wheelchair Use}

There is potential for playful technology to engage wheelchair users in PA: IBM results suggest that wheelchair users place experiential attitude far above the others (indicating a high degree of importance attached to PA among wheelchair users). It is unsurprising that injunctive norm, self-efficacy and perceived control were not positively correlated with the quantity of PA which wheelchair users engage in, because they are most relevant to accessibility (i.e., the built environment, which was repeatedly mentioned as a barrier to accessibility). Similarly, Smith [38] mentions that wheelchairrelated factors and accessibility are associated with participation, which aligns with both personal agency and perceived norm (injunctive norm in particular). Focusing on the importance of positive experiences for wheelchair users while understanding the impact of environmental factors, the remainder of this section reflects on three points for discussion.

(1) Transferring to the Real World: Avoiding Technology Dependency: Our findings suggest a lack of knowledge on how to be physically active, an aspect particularly relevant for individuals transitioning to wheelchair use. Here, games and playful technology offer potential to guide users, supporting them in the development of new PA routines. However, it is important to note that people should be able to engage in PA without depending on technology, suggesting a need for technology-supported PA routines that facilitate scaffolding to technology-free activity. (2) Opening Up New Perspectives on PA Through Play: Following on our findings also show individuals have not previously used technology to engage in PA, opening up new perspectives that could promote regular PA. This shows the importance of playful technology because many participants where excited at the thought of engaging in PA with technology in both our results and other work [10]. (3) Limitations of Games (and Technology in General): Our results show that games in particular appealed to a wide audience, but were strongly rejected by some participants. For those individuals, the combination of PA with games could in fact increase the access barrier to PA rather than lowering it. Likewise, technology can help mitigate some environmental access barriers through design (e.g., awareness of access barriers in local communities when creating mobile systems). However, it is limited to workarounds rather than changing surroundings, suggesting that tangible access barriers need to be addressed on a practical rather than technology level.

\section{Leveraging the IBM for $\mathrm{HCI}$ Requirements Analysis}

Our work demonstrates that the IBM can serve as an effective and agile method of requirements analysis. However, there are two challenges that need to be considered in its application. First, it has not previously been used to study wheelchair users, and only a small number of previous studies have used it within the context of PA (e.g., [5]), making it difficult to relate outcomes to previous studies. Second, the IBM is a tool that provides high-level insights into predictors of behaviour rather than actionable design recommendations. In our work, we added specific questions around technology use and play to both interviews and surveys; then, we tied together IBM results and specific perspectives on technology through the provision of design goals. However, this suggests that the application of the IBM for requirements analysis affords an additional layer of interpretation of our results, suggesting potential in $\mathrm{HCI}$ research rather than as a tool for practitioners that directly leads to actionable outcomes.

\section{LIMITATIONS AND FUTURE WORK}

There are a few limitations that need to be considered when interpreting the findings of our work. The IBM is a newer model in the field of health promotion, which makes it difficult to compare our results with findings from other published applications of the model, requiring individual interpretation. Additionally, the interview study only included eight participants, with an arguably biased demographic (mostly physically active individuals). This needs to be considered when interpreting results from this stage; however, the group of survey respondents was more diverse. Likewise, the survey focuses on quantitative insights. In this context, an in-depth exploration of negative attitudes and barriers to PA and technology use could help us further understand non-engagement with PA and technology. In the future, we plan to leverage our findings to support the development of playful experiences and games aimed to support PA among wheelchair users, exploring stationary versus mobile play, and the impact of social gaming settings on PA participation.

\section{CONCLUSION}

Playful technology has the potential to support PA, but there has been little research regarding the design of playful technology for wheelchair users. Our work provides a mixedmethods enquiry into manual wheelchair users' perspectives on PA and technology, leveraging the IBM to provide an indepth exploration. Findings highlight that anticipation of positive experiences is the strongest predictor of engagement with PA, supporting the development of playful solutions that by nature can highlight this element. Thereby, our research contributes to the growing body of HumanComputer Interaction research addressing the development of technology for people with disabilities that extends beyond accessibility considerations, enabling other researchers and designers to create playful technology that can support PA among wheelchair users. 


\section{REFERENCES}

[1] Shashi K Agarwal. 2012. Cardiovascular benefits of exercise. International journal of general medicine 5 (2012), 541.

[2] Icek Ajzen. 1991. The theory of planned behavior. Organizational behavior and human decision processes 50, 2 (1991), 179-211.

[3] Matthew R Bice, James W Ball, and Steve McClaran. 2016. Technology and physical activity motivation. International fournal of Sport and Exercise Psychology 14, 4 (2016), 295-304.

[4] Jan O Borchers. 2000. A pattern approach to interaction design. In Proceedings of the 3rd conference on Designing interactive systems: processes, practices, methods, and techniques. ACM, 369-378.

[5] Paul Branscum and Amir Bhochhibhoya. 2016. Exploring gender differences in predicting physical activity among elementary aged children: an application of the integrated behavioral model. American Journal of Health Education 47, 4 (2016), 234-242.

[6] Robert E Braun. 2012. Using the integrated behavioral model to predict binge drinking among college students. Ph.D. Dissertation. University of Toledo.

[7] Virginia Braun and Victoria Clarke. 2006. Using thematic analysis in psychology. Qualitative research in psychology 3, 2 (2006), 77-101.

[8] Laurien M Buffart, Tessa Westendorp, Rita J Van Den Berg-Emons, Henk J Stam, and Marij E Roebroeck. 2009. Perceived barriers to and facilitators of physical activity in young adults with childhood-onset physical disabilities. Journal of Rehabilitation Medicine 41, 11 (2009), 881-885.

[9] Bigham Carrington, Laput. [n. d.]. Exploring the Data Tracking and Sharing Preferences of Wheelchair Athletes. ASSETS'18 ([n. d.]).

[10] Patrick Carrington, Kevin Chang, Helena Mentis, and Amy Hurst. 2015. But, I don't take steps: Examining the Inaccessibility of Fitness Trackers for Wheelchair Athletes. In Proceedings of the 17th International ACM SIGACCESS Conference on Computers \& Accessibility. ACM, 193-201.

[11] Valve Corporation. 2007. Portal.

[12] Stephen Cuzzort and Thad Starner. 2008. AstroWheelie: A wheelchair based exercise game. In Wearable Computers, 2008. ISWC 2008. 12th IEEE International Symposium on. IEEE, 113-114.

[13] Antonella De Angeli, Michela Cozza, Mladjan Jovanovic, Linda Tonolli, Mark Mushiba, Andrew McNeill, Lynne Coventry, et al. [n. d.]. Understanding Motivations in Designing for Older Adults.

[14] Martina Eckert, Marcos López, Carlos Lázaro, Juan Meneses, and José F Martínez Ortega. 2015. MoKey-A motion based keyboard interpreter. In Consumer Electronics (ISCE), 2015 IEEE International Symposium on. IEEE, 1-2.

[15] Terry J Ellapen, Henriëtte V Hammill, Mariette Swanepoel, and Gert L Strydom. 2017. The health benefits and constraints of exercise therapy for wheelchair users: A clinical commentary. African fournal of Disability (Online) 6 (2017), 1-8.

[16] inc Fitocracy. 2011. Fitocracy.

[17] Beat Games. 2018. Beat Saber.

[18] Yue Gao, Kathrin M Gerling, Regan L Mandryk, and Kevin G Stanley. 2014. Decreasing sedentary behaviours in pre-adolescents using casual exergames at school. In Proceedings of the first ACM SIGCHI annual symposium on Computer-human interaction in play. ACM, 97-106.

[19] Kathrin M Gerling, Michael R Kalyn, and Regan L Mandryk. 2013. KINECT wheels: wheelchair-accessible motion-based game interaction. In CHI'13 Extended Abstracts on Human Factors in Computing Systems. ACM, 3055-3058.

[20] Kathrin M Gerling, Regan L Mandryk, and Michael R Kalyn. 2013. Wheelchair-based game design for older adults. In Proceedings of the 15th International ACM SIGACCESS Conference on Computers and Accessibility. ACM, 27.
[21] Kathrin Maria Gerling, Matthew Miller, Regan L Mandryk, Max Valentin Birk, and Jan David Smeddinck. 2014. Effects of balancing for physical abilities on player performance, experience and self-esteem in exergames. In Proceedings of the SIGCHI Conference on Human Factors in Computing Systems. ACM, 2201-2210.

[22] Pedro C Hallal, Lars Bo Andersen, Fiona C Bull, Regina Guthold, William Haskell, Ulf Ekelund, Lancet Physical Activity Series Working Group, et al. 2012. Global physical activity levels: surveillance progress, pitfalls, and prospects. The lancet 380, 9838 (2012), 247-257.

[23] Kieran Hicks and Kathrin Gerling. 2015. Exploring casual exergames with kids using wheelchairs. In Proceedings of the 2015 Annual Symposium on Computer-Human Interaction in Play. ACM, 541-546.

[24] Danuta Kasprzyk, Daniel E Montaño, and Martin Fishbein. 1998. Application of an Integrated Behavioral Model to Predict Condom Use: A Prospective Study Among High HIV Risk Groups 1. Journal of Applied Social Psychology 28, 17 (1998), 1557-1583.

[25] Conor Linehan, George Bellord, Ben Kirman, Zachary H Morford, and Bryan Roche. 2014. Learning curves: analysing pace and challenge in four successful puzzle games. In Proceedings of the first ACM SIGCHI annual symposium on Computer-human interaction in play. ACM, 181190.

[26] Ann E Maloney, T Carter Bethea, Kristine S Kelsey, Julie T Marks, Sadye Paez, Angela M Rosenberg, Diane J Catellier, Robert M Hamer, and Linmarie Sikich. 2008. A pilot of a video game (DDR) to promote physical activity and decrease sedentary screen time. Obesity 16, 9 (2008), 2074-2080.

[27] Meethu Malu and Leah Findlater. 2016. Toward accessible health and fitness tracking for people with mobility impairments. In Proceedings of the 10th EAI International Conference on Pervasive Computing Technologies for Healthcare. ICST (Institute for Computer Sciences, Social-Informatics and Telecommunications Engineering), 170-177.

[28] Jeffrey J Martin. 2013. Benefits and barriers to physical activity for individuals with disabilities: a social-relational model of disability perspective. Disability and rehabilitation 35, 24 (2013), 2030-2037.

[29] M Rosly Mat, H Rosly Mat, Nazirah Hasnan, Glen M Davis, and Ruby Husain. 2017. Exergaming boxing versus heavy-bag boxing: are these equipotent for individuals with spinal cord injury? European journal of physical and rehabilitation medicine 53, 4 (2017), 527-534.

[30] Daniel E Montano and Danuta Kasprzyk. 2015. Theory of reasoned action, theory of planned behavior, and the integrated behavioral model. Health behavior: Theory, research and practice (2015), 95-124.

[31] Florian Mueller and Katherine Isbister. 2014. Movement-based game guidelines. In Proceedings of the SIGCHI Conference on Human Factors in Computing Systems. ACM, 2191-2200.

[32] Florian'Floyd' Mueller, Shannon O'Brien, and Alex Thorogood. 2007. Jogging over a distance: Supporting a jogging together experience although being apart. In CHI'07 extended abstracts on Human factors in computing systems. ACM, 1989-1994.

[33] Niantic. 2016. Pokemon GO.

[34] Nintendo. 2007. New Super Mario Bros.

[35] Thomas J OâĂŹConnor, Shirley G Fitzgerald, Rory A Cooper, Tricia A Thorman, and Michael L Boninger. 2002. Kinetic and physiological analysis of the GAME (Wheels) system. F Rehabil Res Dev 39, 6 (2002), 627-634.

[36] Cassandra Phoenix and Noreen Orr. 2014. Pleasure: A forgotten dimension of physical activity in older age. Social science \& medicine 115 (2014), 94-102.

[37] Nora Shields and Anneliese Synnot. 2016. Perceived barriers and facilitators to participation in physical activity for children with disability: a qualitative study. BMC pediatrics 16, 1 (2016), 9.

[38] Emma M Smith, Brodie M Sakakibara, and William C Miller. 2016. A review of factors influencing participation in social and community 
activities for wheelchair users. Disability and Rehabilitation: Assistive Technology 11, 5 (2016), 361-374.

[39] Kevin G Stanley, Ian J Livingston, Alan Bandurka, Mohammad Hashemian, and Regan L Mandryk. 2011. Gemini: A pervasive accumulated context exergame. In International Conference on Entertainment Computing. Springer, 65-76.

[40] Meegan G Van Straaten, Beth A Cloud, Melissa M Morrow, Paula M Ludewig, and Kristin D Zhao. 2014. Effectiveness of home exercise on pain, function, and strength of manual wheelchair users with spinal cord injury: a high-dose shoulder program with telerehabilitation Archives of physical medicine and rehabilitation 95, 10 (2014), 18101817.

[41] Monica Visani Scozzi, Ioanna Iacovides, and Conor Linehan. 2017. A Mixed Method Approach for Evaluating and Improving the Design of Learning in Puzzle Games. In Proceedings of the Annual Symposium on Computer-Human Interaction in Play. ACM, 217-228.

[42] Ross Wadey and Melissa Day. 2018. A longitudinal examination of leisure time physical activity following amputation in England. Psychology of Sport and Exercise 37 (2018), 251-261.

[43] Wouter Walmink, Danielle Wilde, and Florian'Floyd' Mueller. 2014. Displaying heart rate data on a bicycle helmet to support social exertion experiences. In Proceedings of the 8th International Conference on Tangible, Embedded and Embodied Interaction. ACM, 97-104.

[44] Toni Louise Williams, Brett Smith, and Anthony Papathomas. 2014 The barriers, benefits and facilitators of leisure time physical activity among people with spinal cord injury: a meta-synthesis of qualitative findings. Health Psychology Review 8, 4 (2014), 404-425.

[45] Zi Ye, Hamilton A Hernandez, TC Graham, Darcy Fehlings, Lauren Switzer, Md Ameer Hamza, and Irina Schumann. 2012. Liberi and the racer bike: exergaming technology for children with cerebral palsy. In Proceedings of the 14th international ACM SIGACCESS conference on Computers and accessibility. ACM, 225-226. 